\title{
Avaliação do teste de contato com aeroalérgenos em pacientes com dermatite atópica*
}

Evaluation of patch test with airbone allergic agents in patients with atopic der-

\author{
matitis
}

\author{
Rosana Neves Dos Santos Rodrigues ${ }^{1}$ \\ Federico Montealegre ${ }^{3}$ \\ Mario Cezar Pires ${ }^{5}$
}

\author{
João Ferreira Melo ${ }^{2}$ \\ Ruppert Ludwig Hahnstadt ${ }^{4}$
}

\begin{abstract}
Resumo: FunDAMENTOS: a dermatite atópica é uma doença inflamatória cutânea que apresenta múltiplos fatores desencadeantes. Há vários relatos de autores que confirmaram os aeroalérgenos como fatores agravantes ou desencadeantes. O teste de contato com aeroalérgenos ou teste de contato atópico foi proposto para avaliar a participação destes alérgenos na dermatite atópica.

Oвjетіvo: objetivo deste estudo foi avaliar a positividade do teste de contato atópico em pacientes com dermatite atópica.

MÉTodos: Avaliamos 50 pacientes com dermatite atópica e 45 do grupo com rinite alérgica, nos quais realizamos teste de contato atópico com extratos de Dermatophagoides pteronissynus, Dermatophagoides farinae e Blomia tropicalis, além de testes cutâneos de leitura imediata para os mesmos alérgenos, acrescidos de epitélio de cão e gato e fungos.

Resultados: verificamos que o teste de contato atópico com ácaros apresentou maior positividade nos indivíduos do grupo de dermatite atópica quando comparado ao grupo de rinite alérgica.

CONCLuSÕES: o teste de contato atópico apresenta resultados estatisticamente significativos quando realizado com ácaros, em pacientes com dermatite atópica, com $\mathrm{p}=0,035$, OR (odds ratio) $=3,35 \mathrm{e}$ $\mathrm{IC}(95 \%)=[1,18 ; 9,47]$.
\end{abstract}

Palavras-chave: Dermatite atópica; Dermatophagoides pteronyssinus; Hipersensibilidade

\begin{abstract}
BACKGROUND: Atopic dermatitis is an inflammatory skin disease that can be triggered by many factors. Several reports confirm the role of airborne allergic agents as aggravating or triggering factors. The patch test with airborne allergic agents or the atopy patch test was suggested to evaluate the role of these allergens in atopic dermatitis.

ОвлестіvE: This study aimed at evaluating the positivity of the atopy patch test in patients with atopic dermatitis.

Methods: We evaluated 50 patients with atopic dermatitis and 45 with allergic rhinitis, the atopy patch test was performed in these patiennts with extracts of Dermatophagoides pteronissynus, Dermatophagoides farinae and Blomia tropicalis, as well as immediate skin prick tests for the same allergens with cat and dog epithelia and fungi.

RESULTS: It was found that the atopy patch test with dust mites showed higher positivity in individuals with atopic dermatitis when compared to those with allergic rhinitis.

CONCLusions: The atopy patch test shows statistically significant results when performed with dust mites in patients with atopic dermatitis, $p=0.035$, odds ratio $(\mathrm{OR})=3.35$ and CI $(95 \%)=[1.18,9,47]$.

Keywords: Dermatitis, atopic; Dermatophagoides pteronyssinus; Patch tests
\end{abstract}

Recebido em 31.10.2009.

Aprovado pelo Conselho Consultivo e aceito para publicação em 19.04.2010.

* Trabalho realizado no Hospital do Servidor Público Estadual de São Paulo - São Paulo (SP), Brasil

Conflito de interesse: Nenhum / Conflict of interest: None

Suporte financeiro: Nenhum / Financial funding: None

Mestre em Ciências da Saúde - Médica Alergista e Imunologista - São Paulo (SP), Brasil.

Doutor - Diretor do Serviço de Alergia e Imunologia do Hospital do Servidor Público Estadual - São Paulo (SP), Brasil.

Doutor - Professor titular de Imunologia da Escola de Medicina de Ponce - São Paulo (SP), Brasil.

Mestre - Farmacêutico e Diretor do Laboratório FDA-ALLERGENIC - São Paulo (SP), Brasil.

Doutor - Diretor do Serviço de Dermatologia do Complexo Hospitalar Padre Bento de Guarulhos - São Paulo (SP), Brasil. 


\section{INTRODUÇÃO}

A dermatite atópica (DA) é uma dermatose inflamatória crônica e recorrente, caracterizada por lesões eczematosas com localização específica de acordo com a idade e na maioria dos doentes associada à asma e/ou rinite alérgica (RA). ${ }^{1}$

A maior parte dos casos inicia-se na infância, sendo que $60 \%$ surgem durante o primeiro ano de vida e $85 \%$ até os cinco anos de idade ${ }^{1}$. A prevalência dessa doença na cidade de São Paulo está em torno de $13 \%$ nas crianças entre 6 e 7 anos de ambos os gêneros, e na faixa etária de 13 e 14 anos, $12 \%$ no gênero masculino e $15 \%$ no feminino, segundo o estudo ISAAC ("International Study of Asthma and Allergy in Children"). ${ }^{2}$

O quadro clínico cursa com lesões eczematosas agudas, subagudas ou crônicas. No lactente, estão presentes na face e superfície extensora dos membros, na criança maior predominam nas regiões flexoras e no adulto, em locais variados, com predomínio também em flexuras. ${ }^{3}$

Podemos dizer que a DA é uma doença complexa onde interagem a genética, a estrutura própria da pele que é uma característica destes doentes devido a alteração da função de barreira, os distúrbios imunológicos e não imunológicos, sendo vários fatores participantes da sua patogênese. ${ }^{4,5}$

Embora o papel da alergia na patogênese da DA ainda seja controverso sabe-se que em alguns doentes o contato com os aeroalérgenos, entre eles os ácaros, pode desencadear as lesões e vários autores demonstraram evidências da participação destes agentes como desencadeadores das lesões, tanto através de estudos ambientais, como por testes alérgicos. ${ }^{6}$

A relação entre ácaros e DA foi primeiramente observada por Rost* em 1932, ao relatar que alguns pacientes com eczema e também alérgicos a poeira melhoravam do quadro ao serem retirados de suas casas. ${ }^{7}$

Posteriormente outros autores relataram esta relação e observaram em alguns doentes com DA, lesões eczematosas após a aplicação epicutânea de aeroalérgenos, realizada com a técnica do teste de contato modificada e chamada de "atopy patch test" ou teste de contato atópico (TCA). ${ }^{8}$

O TCA é realizado com alérgenos que induzem uma reação IgE mediada em indivíduos portadores de DA. ${ }^{8}$

Sua indicação baseia-se na fisiopatologia da doença, onde foi observado que as células apresentadoras de antígenos (CAA) destes indivíduos têm em sua superfície receptores de alta afinidade para IgE (FceRI) que facilitam a captura e internalização do aeroalérgeno, através da pele. ${ }^{9}$

Associando-se estas características imunológi- cas com a alteração de função da barreira cutânea nestes indivíduos, os aeroalérgenos podem, com maior facilidade, penetrar em direção à epiderme, ligaremse ao IgE-FceRI e depois serem apresentados aos linfócitos T (LT) imaturos provocando sua diferenciação em linfócitos Th2 (LTh2) e posteriormente em Linfócitos Th1 (LTh1). ${ }^{5,6}$

Assim, observando-se as reações imunológicas imediatas e tardias que acontecem nesta doença, foi proposto o TCA para avaliar uma reação cutânea de hipersensibilidade tardia (lesões eczematosas), induzida possivelmente por alérgenos relacionados a reações IgE mediadas. Através do TCA tenta-se reproduzir experimentalmente os eventos fisiopatológicos da DA.

O objetivo deste estudo foi avaliar a positividade do TCA, quando realizado com ácaros (Dpt, Df e $\mathrm{Bt})$, em portadores de DA

\section{PACIENTES, MATERIAIS E MÉTODOS}

$O$ trabalho e o respectivo Termo de Consentimento Livre e Escalrecido (TCLE) foram aprovados pelo Comitê de Ética em Pesquisa do HSPE do IAMSPE e todos os sujeitos da pesquisa, ou responsáveis, assinaram o termo de consentimento para participar do estudo.

Foram selecionados 95 doentes com idade acima de 1 ano, de ambos os sexos, provenientes dos ambulatórios de Dermatologia e Alergia e Imunologia do Hospital do Servidor Público Estadual "Francisco Morato de Oliveira" (HSPE) e do Centro de Alergia da Prefeitura Municipal de Santo André.

Um questionário específico para caracterização da doença foi aplicado em todos os pacientes dos dois grupos. Após aplicação do questionário e exame físico, os doentes foram divididos em dois grupos, segundo o diagnóstico:

A. 50 portadores de DA, com ou sem alergia respiratória (rinite e/ou asma), o diagnóstico estabelecido pelos critérios de Hanifin e Rajka ${ }^{10}$ e a gravidade pelo SCORAD ${ }^{11}$ ("severity score of atopic dermatitis").

B. 45 com diagnóstico de RA, estabelecido segundo O ARIA (ALLERGIC RHINITIS AND ITS IMPACT ON ASTHMA), ${ }^{12}$ com ou sem asma, mas sem evidências de doença dermatológica. O diagnóstico de asma baseou-se no III Consenso Brasileiro no Manejo da Asma, 2002. ${ }^{13}$

Foram realizados hemograma, protoparasitológico em três amostras, dosagem de IgE total, teste cutâneo de leitura imediata para ácaros (Dpt, Df e Bt), epitélio de cão e gato, Alternaria alternata e fungos III ( Aspergillus astelodami, niger e terreus) e IV (Peniccilinum brevicompactum, expansum e notatum). 
O TCA com extrato padronizado de teste cutâneo de leitura imediata para Dpt, Df, Bt e o diluente dos mesmos foi aplicado em todos os pacientes.

\section{Critérios de inclusão:}

q sujeitos da pesquisa que ele ou seus responsáveis legais leram e assinaram o termo de consentimento livre e esclarecido.

q Grupo A - Pacientes com DA com idade $\geq 1$ ano, com ou sem alergia respiratória.

q Grupo B - Pacientes com RA, com ou sem asma, e sem doenças de pele.

\section{Critérios de exclusão:}

1. Portadores de outras doenças dermatológicas exceto DA em ambos os grupos;

2. Portadores de rinite não alérgica;

3. Gravidez;

4. Idade inferior a um ano;

5. Doenças graves;

6. Pacientes em uso de imunossupressores;

7. Pacientes utilizando corticosteróides sistêmicos e/ou tópicos no local dos testes e outros medicamentos que pudessem interferir na realização dos mesmos.

8. Sujeitos da pesquisa incapazes de ler ou compreender o termo de consentimento livre e esclarecido.

9. Aqueles que se recusaram a assinar o TCLE.

Os extratos para o TCA(Dpt, Df e Bt) e o seu diluente, foram preparados a partir do extrato de teste cutâneo de leitura imediata diluídos a 30\%, utilizando-se vaselina sólida como veículo. Estes foram preparados e cedidos pelo laboratório FDA ALLERGENIC.

A técnica de aplicação do TCA foi realizada seguindo a padronização do Departamento Especializado de Alergia em Dermatologia, da Sociedade Brasileira de Dermatologia (SBD). ${ }^{14}$

Os testes foram aplicados em câmaras de alumínio de $8 \mathrm{~mm}$ na parte superior da região dorsal dos pacientes, previamente limpa com éter. Imediatamente antes do teste, fez-se abrasão com fita de micropore dez vezes no mesmo local, com a finalidade de facilitar a absorção dos antígenos, conforme descrito em outros estudos. ${ }^{15}$

As substâncias, com distância de cinco centímetros uma da outra, foram deixadas no dorso por 48 horas e então retiradas (dia 2 ). A segunda leitura aconteceu 96 horas após a colocação do teste (dia 4) (Figura 1), seguindo os critérios estabelecidos pelo Grupo Brasileiro de Estudos de Dermatite de Contato (GBEDC). ${ }^{14}$

(-) negativo

(?) duvidoso

(+) eritema com algumas pápulas
$(++)$ eritema, pápulas e vesículas

$(+++)$ intenso eritema, pápulas e vesículas confluentes.

Foram considerados como resultados positivos somente as reações dos testes que foram iguais ou superiores na sua intensidade, na leitura de 96 horas em relação à de 48 horas, portanto aquelas que tiveram um aspecto em "crescendo". As reações que apresentaram menor intensidade em 96 horas quando comparadas às de 48 horas, consideramos irritativas e não foram relevantes para o estudo.

Os extratos comercialmente disponíveis para realizar o teste cutâneo de leitura imediata de Dpt e Df, epitélio de cão e gato, alternaria alternata e fungos III ( Aspergillus astelodami, niger e terreus) e IV (Peniccilinum brevicompactum, expansum e notatum), foram cedidos pelo laboratório ALK ABELLÓ e o deBt, pela IPI - ASAC Brasil.

Esse teste foi realizado segundo a técnica de Pepys, pela via percutânea (puntura), na superfície volar do antebraço. Após limpeza da região com álcool a $70^{\circ} \mathrm{GL}$ foram colocadas uma gota de cada um dos extratos acima citados, do diluente (controle negativo) e da histamina (controle positivo). Os dispositivos usados foram puntores descartáveis, um para cada substância, introduzidos perpendicularmente à superfície cutânea, exercendo leve pressão sobre cada gota e a leitura realizada após 20 minutos do início do teste pela medida do diâmetro médio da pápula. Foram consideradas positivas as reações maiores ou iguais a 3 milímetros de diâmetro médio para cada substância. ${ }^{16}$

Foram suspensos os medicamentos que pudessem interferir no resultado do teste, sendo que a orientação foi a de interromper o uso de antihistamínicos 10 dias antes da realização do teste cutâneo de leitura imediata. ${ }^{16}$

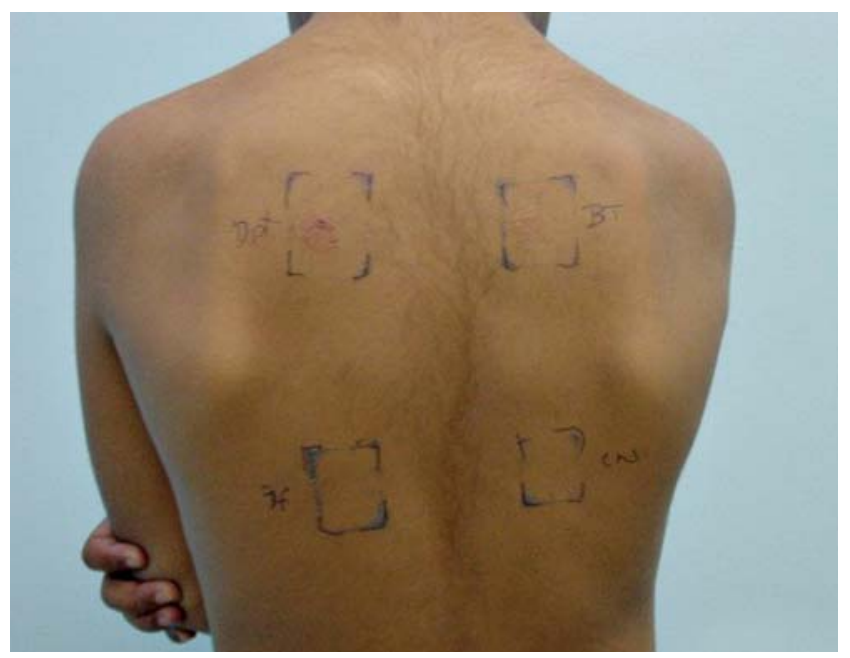

Figura 1: Resultado do Teste de Contato Atópico positivo para Dermatophagoides Pteronyssinus 


\section{RESULTADOS}

Dos 95 pacientes avaliados, 53\% apresentava DA diagnosticada, enquanto $48 \%$ tinham RA.

No grupo com DA a mediana da idade foi de 4,9 anos e de 9,3 anos no de RA. No grupo de DA, 31 dos doentes (62\%) pertenciam ao gênero feminino e 19 ao masculino (38\%), enquanto que no grupo de RA, $13(28,8 \%)$ eram do gênero feminino e $32(71,1 \%)$ do gênero masculino.

No grupo da DA, 17 (34\%) de 50 pacientes apresentaram pelo menos um TCA positivo, enquanto naqueles com RA, houve $6(13,3 \%)$ de 45 doentes com positividade em pelo menos um TCA (Tabela 1).

Não houve reações positivas nos testes realizados com o diluente em ambos os grupos.

Foram encontradas diferenças estatisticamente significativas entre os resultados para os grupos DA e RA $(\mathrm{p}<0,05)$.

A chance deste teste ser positivo no grupo de DA foi 3,35 vezes maior em relação ao grupo RA.

Os resultados mostraram diferenças estatisticamente significativas entre os dois grupos com $\mathrm{p}<0,05$.

A OR foi de 2,5, mostrando maior chance do paciente com DA ter o TCA positivo.

Em relação ao número total de testes positivos em cada grupo, encontramos $25(16,7 \%)$ positivos no grupo DA e $10(7,4 \%)$ no de RA. O total de testes positivos mostrou maior relevância no grupo da DA em relação aqueles com RA, o que foi estatisticamente significativo $(p=0,017)$. (Tabela 2$)$

Dos 50 pacientes do grupo de DA, 42 (84\%) apresentaram teste cutâneo de leitura imediata positivo para no mínimo um alérgeno e dos 17 doentes do mesmo grupo com pelo menos um teste de contato atópico positivo, 16 (94\%) também o tiveram no teste cutâneo de leitura imediata. Destes 16 indivíduos, 12 (75\%) obtiveram reação positiva ao mesmo alérgeno nos dois testes.

\section{Métodos estatísticos}

Os resultados dos TCA entre os grupos de DA e RA foram representados através de tabelas de contin- gência e a comparação entre os diagnósticos, quanto aos resultados dos testes, pelo teste qui-quadrado de Pearson com correção de continuidade. Foi calculada a OR (odds ratio), ou razão de chances, e o respectivo IC - 95\% (intervalo com 95\% de confiança) para a OR.

O nível de significância adotado foi de $0,05(\alpha$ $=5 \%)$ e níveis descritivos (p) inferiores a esse valor foram considerados significantes.

\section{DISCUSSÃO}

A DA é uma doença complexa onde estão envolvidos vários fatores desencadeantes ou agravantes, intrínsecos e extrínsecos, dentre estes, destacamos os aeroalérgenos. ${ }^{17}$ Muitos pacientes apresentam teste cutâneo de leitura imediata positivo para aeroalérgenos. Estes testes apresentam boa relevância em relação às doenças alérgicas respiratórias, onde o papel dos aeroalérgenos está bem estabelecido. A relação destes na DA ainda é controversa, pois esta doença cursa com quadro eczematoso que condiz com uma reação tardia mediada por linfócitos. Estes exames avaliam a reação imediata que não representa a manifestação clínica da DA e também não avaliam por completo a resposta imune cutânea desta doença. ${ }^{18}$

A pele está exposta às influências do meio ambiente, incluindo os alérgenos, e postula-se que estes podem aderir à sua superfície e de forma repetida induzir sensibilização ou elicitação através deste órgão, baseados na fisiopatologia da doença. ${ }^{19}$

Todos estes eventos estimularam alguns autores a tentar reproduzir as lesões da dermatite atópica através da provocação com fatores desencadeantes, como os ácaros da poeira domiciliar. Como na DA as lesões são eczematosas, instituiu-se um modelo de estudo semelhante aquele da dermatite alérgica de contato. $^{8}$

O primeiro trabalho com TCA foi publicado em 1982 por Mitchell et al e posteriormente outros foram realizados com metodologias diferentes, utilizando-se vários veículos e concentrações, assim como algum tipo de abrasão na pele, na tentativa de padronizá-los. ${ }^{20}$

Em um estudo Goon observou uma positividade de $43,9 \%$ para o ácaro Dermatophagoides pte-

TABela 1: Pacientes que apresentaram no mínimo um TCA positivo, em relação ao diagnóstico de DA ou RA na leitura de 96 horas

\section{Diagnóstico}

\begin{tabular}{llll}
\cline { 2 - 4 } Resultado TCA & Dermatite Atópica & Rinite & Total \\
\hline Positivo & $17(34,0 \%)$ & $6(13,3 \%)$ & $23(24,2 \%)$ \\
Negativo & $33(66,0 \%)$ & $39(86,7 \%)$ & $72(75,8 \%)$ \\
Total & $50(100 \%)$ & $45(100 \%)$ & $95(100 \%)$ \\
X2 $=4,44$ & $\mathrm{p}=0,035$ & OR $=3,35$ & IC $(95 \%)=[1,18 ; 9,47]$ \\
\hline
\end{tabular}


TABela 2: Número total de testes positivos segundo o diagnóstico de DA ou RA

\begin{tabular}{llll}
\hline & & Diagnóstico & \\
\cline { 2 - 4 } Resultado TCA & Dermatite Atópica & Rinite Alérgica & Total \\
\hline Positivo & $25(16,7 \%)$ & $10(7,4 \%)$ & $35(12,3 \%)$ \\
Negativo & $125(83,3 \%)$ & $125(92,6 \%)$ & $250(87,7 \%)$ \\
Total & $150(100 \%)$ & $135(100 \%)$ & $285(100 \%)$ \\
X2 $=4,83$ & $\mathrm{p}=0,028$ & OR $=2,50$ & IC $(95 \%)=[1,09 ; 5,84]$ \\
\hline
\end{tabular}

X2 (com correção de continuidade $)=4,83$

$\mathrm{p}=0,028$

ronyssinus, assim como uma diferença estatisticamente significativa quando comparou estes testes nos doentes com DA e RA . ${ }^{21}$

Jamora et al realizaram TCA com uma mistura de Dpt $50 \%$ e Df $50 \%$ diluídos em vaselina a $20 \%$. Esta diluição foi fracionada em concentrações equivalentes a $1,25 \% ; 1,0 \%, 0,75 \% ; 0,5 \%, 0,25 \%$ e $0,1 \%$. Em seus resultados, demonstraram que a concentração de $0,1 \%$ foi melhor para positividade do TCA em pacientes com DA, enquanto que nas outras a positividade foi semelhante aos grupos de alergia respiratória e indivíduos não alérgicos. ${ }^{22}$

Outros estudos com TCA foram feitos para se avaliar a sua positividade assim como a sua reprodutibilidade. Ingordo observou maior positividade deste teste no grupo da DA quando comparado aos indivíduos não alérgicos e com alergia respiratória. $O$ mesmo teste foi repetido em alguns pacientes dos três grupos e apresentou boa reprodutibilidade. ${ }^{23}$

Em nosso estudo, realizamos TCA com três tipos de ácaros (Dpt, Df e Bt) em pacientes com DA associada ou não com alergia respiratória e em portadores de RA sem doença dermatológica. Optamos pela vaselina como veículo, baseados em dados de literatura. ${ }^{20}$

Ao analisarmos o número total de TCA positivos realizados com os 3 ácaros (Tabela 2), foram encontradas diferenças estatisticamente significativas $(p=0,028)$, assim como quando avaliamos o número de pacientes que apresentou pelo menos um TCA positivo no grupo da DA em relação ao da RA (tabela $1, \mathrm{p}=0,035)$.

Estes resultados demonstram associação significativa entre a exposição de ácaros e o desencadeamento das lesões eczematosas, o que justifica o relato de pacientes quando dizem piorar após o contato com estes alérgenos.

Justifica também que a DA pode preceder a alergia respiratória, o que é chamado de marcha atópica, conforme alguns estudos tentam demonstrar, pelo fato de que a sensibilização pode ter início na pele devido às alterações da função de barreira cutânea que facilitam a penetração destes alérgenos e o desen- cadeamento da resposta imune. A partir da diferenciação de LT maduros em LTh2, uma parte destes vai migrar pela circulação sanguínea para outros locais, como a mucosa nasal e pulmonar. ${ }^{5,19}$

Futuramente, o contato com o mesmo alérgeno poderá provocar reação sistêmica clinicamente demonstrada por quadros de asma, rinite e piora da DA. ${ }^{19}$

A diminuição da função de barreira cutânea é a "marca" da DA e esta normalmente é a primeira manifestação de doença alérgica na infância, seguida pelo desenvolvimento de doenças respiratórias alérgicas, como asma e rinite. Estas doenças têm características em comum e aproximadamente $60 \%$ das crianças com DA apresentam asma e 35\% das mesmas, rinite.,

A região cromossômica relacionada com a DA difere daquela que tem relação com as doenças alérgicas respiratórias, fato que sugere alterações genéticas diferentes. Assim, a etiologia comum que pode promover a ocorrência destas doenças em um mesmo indivíduo, seria a alteração da barreira cutânea que acontece devido a uma mutação genética ${ }^{51,8}$ e assim os alérgenos, as substâncias irritantes e os microorganismos, podem através da pele, induzir respostas imunológicas. ${ }^{5,25,26}$

A ocorrência destas respostas está amparada em estudos prévios que demonstram características imunológicas em comum aos dois grupos de doenças, DA e alergia respiratória, assim como na observação da maior positividade do TCA em portadores de DA quando comparados aos indivíduos com alergia respiratória e não alérgicos, fato que reforça a possibilidade do comprometimento funcional da barreira cutânea nestes indivíduos. ${ }^{9,27}$

Portanto, podemos dizer que a relação dos aeroalérgenos, entre eles, os ácaros, com a DA é relevante e deve ser considerada no acompanhamento destes doentes, pois como eles apresentam alteração da função de barreira cutânea, a penetração destes agentes é facilitada.

A realização do TCA e a sua positividade em parte dos indivíduos com DA, mostra que além da relação com os aerolalérgenos, outros antígenos 
como alimentos, microorganismos podem participar da fisiopatologia da DA, colaborando com a manutenção ou desencadeamento das lesões. ${ }^{28,29}$

Pode também nos mostrar que um mesmo antígeno é capaz de induzir reações imunológicas diferentes em um mesmo indivíduo, como pudemos observar nos 12 pacientes que apresentaram reação positiva aos mesmos alérgenos nos dois testes. Com estes dados observamos que o TCA é um instrumento que pode colaborar na elucidação de agentes desencadeantes da DA assim como na avaliação da sua fisiopatologia. ${ }^{29}$

Por outro lado, os trabalhos da literatura nos mostram que este teste não é um método padronizado, necessita ainda de mais pesquisas no sentido de buscar a melhor sensibilidade e especificidade para cada alérgeno. ${ }^{30}$

É sabido que a alergia não é o único fator etiológico desta doença, assim como a hipersensibilida- de não é o único fator que contribui para a positividade do TCA, mas este teste poderá identificar a sensibilização aos ácaros da poeira doméstica, que têm a possibilidade de estimular a reação imunológica tardia, associada ao desencadeamento ou piora das lesões eczematosas na DA, e sustenta o conceito de que o IgE e os linfócitos T estão envolvidos na fisiopatologia desta doença, assim como o conceito de que a DA não é somente uma doença com disfunção de barreira cutânea e pele seca, mas também uma doença alérgica. ${ }^{18}$

Tendo em vista os resultados obtidos neste estudo e os observados na literatura, consideramos que o TCA tem relação importante com a DA, mas que precisa ser mais estudado.

\section{CONCLUSÕES}

Observamos que a positividade do TCA foi maior nos pacientes com DA quando comparada aos pacientes com RA. 


\section{REFERÊNCIAS}

1. Rios JB, Carvalho LP, Martins ER, Emerson FE, Tebyriça JN. Dermatite Atópica. In: Rios JB, Carvalho LP, Martins ER, Emerson FE, Tebyriça JN. Alergia Clínica Diagnóstico e Tratamento. Rio de Janeiro: Revinter; 1995. p. 193-201.

2. Yamada E, Vanna AT, Naspitz CK, Solé D. International Study of Asthma and Allergies in Chidhood (ISAAC): validation of the written questionnaire (eczema component) and prevalence of atopic eczema among Brazilian Children. J Investig Allergol Clin Immunol. 2002;12:34-41.

3. Beltrani VS. The clinical spectrum of atopic dermatitis. J Allergy Clin Immunol. 1999;104:S87-98.

4. Leung DY, Bieber T. Atopic dermatitis. Lancet. 2003;361:151-60.

5. Hoffjan S, Stemmler S. On the role of epidermal differentiation complex in ichthyosis vulgaris, atopic dermatitis and psoriasis. Br J Dermatol. 2007;157:441-9.

6. Leung DY, Boguniewicz M, Howell MD, Nomura I, Hamid QA. New insights into atopic dermatitis. J Clin Invest. 2004;113:651-7.

7. Platts-Mills TA, Mitchell EB, Rowntree S, Chapman MD, Wilkins SR. The role of dust mite allergens in atopic dermatitis. Clin Exp Dermatol. 1983;8:233-47.

8. Clark RA, Adinoff AD. Aeroallergen contact can exacerbate atopic dermatitis: patch tests as a diagnostic tool. J Am Acad Dermatol. 1989;21:863-9.

9. von Bubnoff D, Novak N, Kraft S, Bieber T. The central role of FcepsilonRI in allergy. Clin Exp Dermatol. 2003:28:184-7.

10. Hanifin JM, Rajka G. Diagnostic features of atopic dermatitis. Acta Derm Venereol. 1980:92:\$44-7.

11. Severity scoring of atopic dermatitis: the SCORAD index. Consensus Report of the European Task Force on Atopic Dermatitis. Severity scoring of atopic dermatitis: the SCORAD index. Dermatology. 1993;186:23-31.

12. Bousquet J, Van Cauwenberge P, Khaltaev N, Aria Workshop Group, World Health Organization. Allergic rhinitis and its impact on asthma. J Allergy Clin Immunol. 2001;108(5 Suppl):S147-334.

13. III Consenso Brasileiro no Manejo da Asma 2002. J Pneumol. 2002;28(S1):S128.

14. Grupo Brasileiro de Estudo em Dermatite de Contato (GBEDC); Sociedade Brasileira de Dermatologia. Departamento Especializado de Alergia. Estudo multicêntrico para elaboração de uma bateria-padrão brasileira de teste de contato. An Bras Dermatol.2000;75:147-56.

15. Fernandes MF, Mello JF, Pires MC, Vizeu MC. Comparative study of patch test using traditional method vs. prior skin abranding. J Eur Acad Dermatol Venereol. 2007;21:1351-9.

16. Demoly P, Mitchel FB, Bousquet J. In Vivo methods for Study of Allergy Skin Tests, Techniques, and Interpretation. In: Middleton EJ, Reed CE, Ellis EF, Adkinson NF, Yunginger JW, Busse WW. Allergy Principles e Practice. St Louis: Mosby; 1998. p.430-9.

17. Beck HI, Korsgaard J. Atopic dermatitis and house-dust mites. Acta Derm Venereol Suppl (Stockh).1989;144:125-6.

18. Darsow U, Ring J. Immunoglobulin e-mediated allergy plays a role in atopic eczema as shown in the atopy patch test. World Allergy Organiz J. 2008;1:51-6.
19. Beck LA, Leung DY. Allergen sensitization through the skin induces systemic allergic responses. J Allergy Clin Immunol. 2000;106(5 Suppl):S258-63.

20. Darsow U, Vieluf D, Ring J. Atopy patch test with different vehicles and allergen concentrations: an approach to standardization. J Allergy Clin Immunol. 1995:95:677-84

21. Goon A, Leow YH, Chan YH, Ng SK, Goh CL. Atopy patch testing with aeroallergens in patients with atopic dermatitis and controls in Singapore. Clin Exp Dermatol. 2005;30:627-31.

22. Jamora MJ, Verallo-Rowell VM, Samson-Veneracion MT. Patch Testing with 20\% Dermatophagoides pteronyssinus/farinae (Chemotechnique) antigen. Am J Contact Dermat. 2001;12:67-71.

23. Ingordo V, Dalle Nogare R, Colecchia B, D'Andria C. Is the atopy patch test with house dust mites specific for atopic dermatitis? Dermatology. 2004;209:276-83.

24. Cork MJ, Robinson DA, Vasilopoulos Y, Ferguson A, Moustafa M, MacGowan A, et al. New perspectives on epidermal barrier dysfunction in atopic dermatitis: geneenvironment interactions. J Allergy Clin Immunol. 2006;118:3-21.

25. Marenholz I, Nickel R, Rüschendorf F, Schulz F, Esparza-Gordillo J, Kerscher T, et al. Filaggrin loss-of-function mutations predispose to phenotypes involved in the atopic march. J Allergy Clin Immunol. 2006;118:866-71.

26. Callard RE, Harper JI. The skin barrier, atopic dermatitis and allergy: a role for Langerhans cells? Trends Immunol. 2007;28:294-8.

27. Turjanmaa $\mathrm{K}$. The role of atopy patch tests in the diagnosis of allergy in atopic dermatitis. Curr Opin Allergy Clin Immunol. 2005:5:425-8.

28. Baker BS. The role of microorganisms in atopic dermatitis. Clin Exp Immunol 2006:144:1-9.

29. Samochocki Z, Owczarek W, Rujna P, Raczka A. Hypersensitivity to aeroallergens in adult patients with atopic dermatitis develops due to the different immunological mechanisms. Eur J Dermatol. 2007;17:520-4.

30. Turjanmaa K, Darsow U, Niggemann B, Rancé $F$, Vanto T, Werfel T. EAACI/GA2LEN position paper: present status of the atopy patch test. Allergy. 2006;61:1377-84.

\author{
ENDEREÇO PARA CORRESPONDÊNCIA / MAILING ADDRESS: \\ Rosana Neves dos Santos Rodrigues \\ Rua Xingu - 245 AP 12, Valparaiso \\ 09060050 - Santo André - SP, Brasil \\ Tel.: 1199375755
}

Email:rosana.neves07@gmail.com

Como citar este artigo/How to cite this article: Morais PM, Cunha MGS, Frota MZM. Aspectos clínicos de pacientes com pitiríase versicolor atendidos em um centro de referência em dermatologia tropical na cidade de Manaus -Amazonas, Brasil. An Bras Dermatol. 2011;86(1):37-43. 